\title{
THE GRAVITATIONAL SHOCK WAVE OF a MASSLESS PARTICle
}

\author{
Tevian DRAY' and Gerard 't HOOFT \\ Instituut voor Theoretische Fysica, Princetonplein 5, Postbus 80.006, 3508 TA Utrecht, The Netherlands
}

Received 20 August 1984

\begin{abstract}
The (spherical) gravitational shock wave due to a massless particle moving at the speed of light along the horizon of the Schwarzschild black hole is obtained. Special cases of our procedure yield previous results by Aichelburg and Sexl [1] for a photon in Minkowski space and by Penrose [2] for sourceless shock waves in Minkowski space. A new derivation of the (plane) shock wave of a photon in Minkowski space [1] involving explicit calculation of geodesics crossing the shock wave is also given in order to clarify the underlying physics. Applications to quantum gravity, specifically the possible effect on the Hawking temperature, are briefly discussed.
\end{abstract}

\section{Introduction}

There are various reasons why one may be interested in exact expressions for the gravitational field surrounding a particle whose mass is dominated by kinetic energy rather than rest mass. For instance the first non-trivial gravitational effects to be seen in particle-particle interactions at extreme energies may be due to such fields. Our understanding of quantum gravity may be helped by considering these field configurations. A specific case of interest is the gravitational back-reaction and self-interaction of matter entering or leaving a black hole (Hawking radiation). At the black hole horizon the relative velocity of these particles approach that of light.

Aichelburg and Sexl [1] considered the gravitational field of a massless particle in Minkowski space, and showed that the resulting space-time is a special case of a gravitational impulsive wave ${ }^{\star}$ [2] which is also an asymmetric plane-fronted gravitational wave [3]. Penrose [2] also gives explicit examples of sourceless gravitational impulsive waves in Minkowski space.

In this paper we first summarize the properties of the shock wave due to a massless particle in Minkowski space. We do this by presenting a new derivation of the results of Aichelburg and Sexl [1] involving explicit calculation of (null) geodesics crossing the shock wave. This enables the physical properties of such shock waves to be easily exhibited.

We then determine, for a particular class of vacuum solutions to the Einstein field equations, the (necessary and sufficient) conditions for being able to introduce

1 Supported by the Stichting voor Fundamenteel Onderzoek der Materie.

* Note that Penrose [2] reserves the term gravitational shock wave for a metric which is $\mathrm{C}^{1}$ whereas the metrics we consider are only $\mathrm{C}^{0}$. We will nevertheless use the term "shock wave" for what are, in the terminology of [2], impulsive waves. 


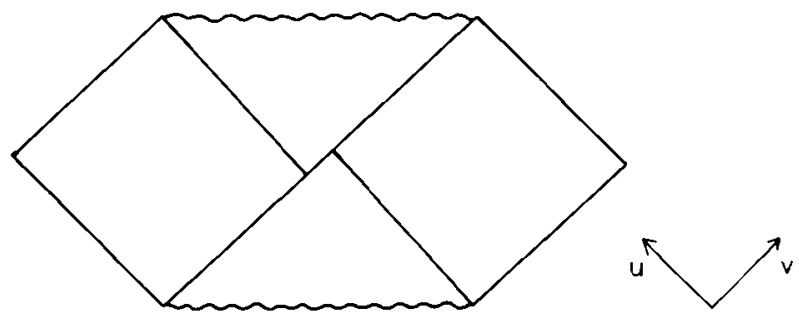

Fig. 1. The horizon shift (eq. (15)) due to the field of a massless particle moving in the $v$-direction along the horizon of the Schwarzschild black hole. The amount of the shift depends on $\theta$.

a gravitational shock wave via a coordinate shift ${ }^{\star}$. These conditions include both constraints on the metric coefficients and on the form of the shift. In Minkowski space they reduce to the plane-fronted wave of Aichelburg and Sexl [1] and, of course, to Penrose's results [2] for sourceless waves. However, for Schwarzschild black holes we obtain something new: there is a (spherical) shock wave at the horizon due to a massless particle at the horizon. (See fig. 1.)

Throughout this paper we think of the massless particle as the limit of a fast-moving particle with negligible rest mass ${ }^{\star \star}$; this limit is given explicitly for the Minkowski case. Fig. 1 can thus be interpreted as describing an ordinary particle with small mass falling into the black hole from the left, as seen by an outside observer (on the left) at very late times; the particle is then seen close to the horizon and boosted to high energies.

The paper is organized as follows: in sect. 2 we summarize the situation for the (plane) shock wave due to a massless particle in Minkowski space and discuss the general physical features of such a wave. These results are based on a calculation of the null geodesics in such a space-time, which is given explicitly in appendix $\mathrm{A}$. In sect. 3 we give the conditions, derived in appendix B, for a shock wave to be possible starting from a given "background" space-time. After showing that these conditions reduce to the correct ones $[1,2]$ in Minkowski space we then obtain the (spherical) shock wave at the horizon of the Schwarzschild black hole due to a massless particle there. In sect. 4 we discuss our results.

\section{Shock waves: an example}

Aichelburg and Sexl [1] (cf. eq. (A.37)) have shown that the gravitational field of a massless particle in Minkowski space is described by the metric

$$
\mathrm{d} s^{2}=-\mathrm{d} \hat{u}\left(\mathrm{~d} \hat{v}+4 p \ln \left(\rho^{2}\right) \delta(\hat{u}) \mathrm{d} \hat{u}+\mathrm{d} x^{2}+\mathrm{d} y^{2},\right.
$$

* This is just the scissors-and-paste approach of Penrose [2] applied to more general space-times.

* We assume that the particle has no electric charge and no angular momentum. However, for an elementary particle for example we do not expect the results to differ significantly from those we derive here. 
where $\rho^{2}=x^{2}+y^{2}$. The particle moves in the $\hat{v}$ direction with momentum $p$. By calculating geodescis which cross the shock wave, which is located at $u=0$, we obtain the following two physical effects of such a shock wave (see appendix $A$ ): geodescis have a discontinuity $\Delta \hat{v}$ at $u=0$ and are refracted in the transverse direction. The shift $\Delta \hat{v}$ is given by (cf. eq. (A.26))

$$
\Delta \hat{v}=-\frac{4 G p}{c^{3}} \ln \frac{\rho_{0}^{2}}{l_{\mathrm{P} \mid}^{2}},
$$

which, for a photon, is

$$
\Delta \hat{v}=-\frac{4 l_{\mathrm{Pl}}^{2} \nu}{c} \ln \frac{\rho_{0}^{2}}{l_{\mathrm{Pl}}^{2}},
$$

where we have put the units back in and where we have used $E=p c=\hbar \nu$, where $\nu$ is the frequency of the photon and $l_{P \mid}$ is the Planck length. $\rho_{0}$ is the value of $\rho$ when the geodesic reaches $u=0$. This shift is illustrated (for nonzero $m$ and $x=0$ ) in fig. 2; for $m=0$ the shift occurs as a discontinuity at $u=0$.

Note that the presence of a length scale in the argument of the logarithm is merely a reflection of our choice of units and has no physical meaning. It represents a constant shift in $\hat{v}$ which can be transformed away by a suitable redefinition of $\hat{v}$ (eq. (A.11)). Furthermore, by the same procedure, the value of $\rho_{0}$ for which $\Delta \hat{v}=\mathbf{0}$ (here $\rho_{0}=l_{p l}$ ) can be chosen arbitrarily far from the photon ( $\rho_{0}$ large). In any case, only the difference in $\Delta \hat{v}$ for nearby geodesics is physically relevant.

There is also a refraction effect described by (cf. eq. (A.36))

$$
\cot \alpha+\cot \beta=\frac{4 G p}{c^{3} \rho_{0}},
$$

which, for a photon, is

$$
\cot \alpha+\cot \beta=\frac{4 l_{\mathrm{P} 1}^{2} \nu}{c \rho_{0}} .
$$

This is illustrated (for $x=0$ ) in fig. 3, where the angles $\alpha$ and $\beta$ are defined.

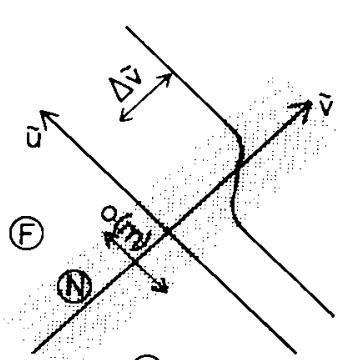

(F)

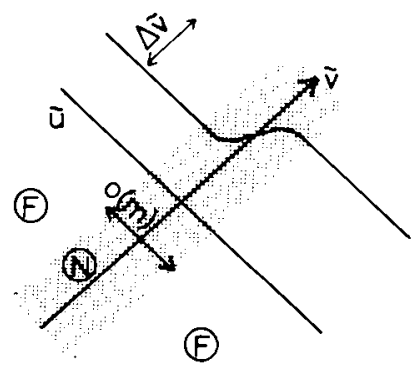

b) $y_{0}>1$

Fig. 2. The path of a null geodesic in the $(\hat{u}, \hat{v})$ plane as described by eq. (2) for $m<1, \rho_{0}>m$, and (a) $\rho_{0}<1$, (b) $\rho_{0}>1$. The near region $N$ and the far region $F$, as well as the shift $\Delta \hat{v}$, are indicated. 


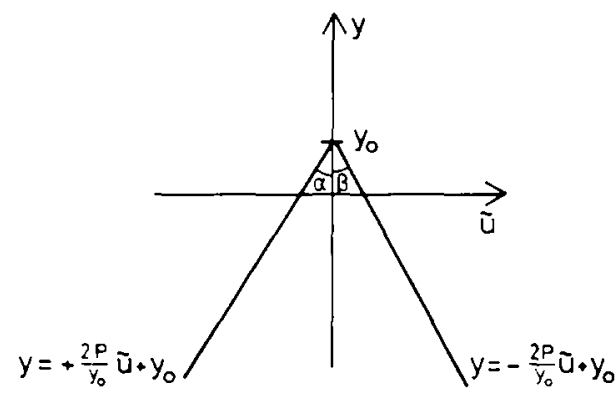

(a)

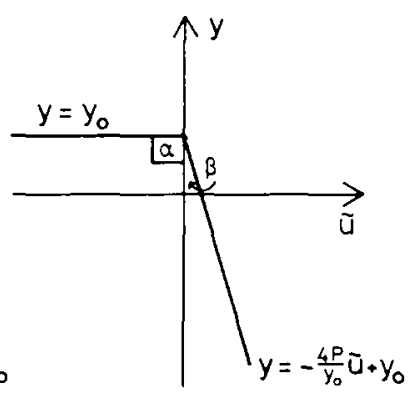

(b)

Fig. 3. The "spatial refraction" of null geodesics as described by eq. (3) for the two special cases (a) $\alpha=\beta$, and (b) $\alpha=\frac{1}{2} \pi$.

Eqs. (2) and (3) are the central results for these shock waves and describe physical effects which should also occur in more general situations. Note that if the shift (2) were constant it could be removed by a coordinate translation and would therefore not be physically observable (cf. the discussion after eq. (2)). Also, a shift linear in the transverse distance $\rho$ would not be observable since it could be removed by a Lorentz rotation of one of the flat half-spaces with respect to the other. However the shift (2) is logarithmic in $\rho$ and leads to physically observable effects. The relative shift for nearby observers goes as the first derivative $(1 / \rho)$ while the relative refraction goes as the second derivative $\left(1 / \rho^{2}\right)$ of the shift ${ }^{\star}$. See fig. 4 .

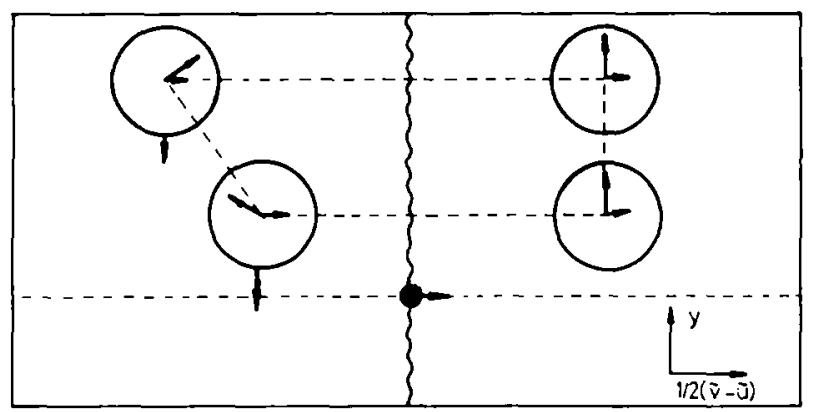

Fig. 4. Four synchronized clocks were originally situated at rest at the corners of a rectangle. A fast particle approaches from the left. The situation is shown when the shock wave has passed two of the clocks. The one closest to the trajectory of the particles has been shifted to the right with respect to the other; its clock now runs behind the other. They are also moving towards the trajectory of the fast particle at different speeds (arrows). Only their relative velocity, which is always away from each other, is locally observable.

* Note that a local observer can only detect the second derivative $\left(1 / \rho^{2}\right)$ of the shift. 


\section{General result}

Consider a solution of the vacuum Einstein field equations of the form

$$
\mathrm{d} \hat{s}^{2}=2 A(u, v) \mathrm{d} u \mathrm{~d} v+g(u, v) h_{i j}\left(x^{i}\right) \mathrm{d} x^{i} \mathrm{~d} x^{j} .
$$

Under what conditions can we introduce a shift in $v$ at $u=0$ so that the resulting space-time solves the field equation with a photon at the origin $\rho=0$ of the $\left(x^{i}\right)$ 2-surface and $u=0$ ? As shown in appendix $B$ the answer is the at $u=0$ we must have

$$
\begin{gathered}
A_{v v}=0=g_{, v}, \\
\frac{A}{g} \Delta f-\frac{g_{\text {uv }}}{g} f=32 \pi p A^{2} \delta(\rho),
\end{gathered}
$$

where $f=f\left(x^{i}\right)$ represents the shift in $v, \Delta f$ is the laplacian of $f$ with respect to the 2-metric $h_{i j}$, and the resulting metric is described by (B.2) or (B.4). Eqs. (5) represent our main result. We now turn to specific examples.

For a plane wave due to a photon in Minkowski space we have

$$
\mathrm{d} \hat{s}^{2}=-\mathrm{d} u \mathrm{~d} v+\mathrm{d} x^{2}+\mathrm{d} y^{2},
$$

and thus

$$
\begin{aligned}
& \boldsymbol{A}=-\frac{1}{2}, \\
& \boldsymbol{g}=1 .
\end{aligned}
$$

The conditions on the metric are trivially satisfied, and the condition on the shift $f$ is

$$
\Delta f=-16 \pi p \delta(\rho),
$$

where $\rho^{2}=x^{2}+y^{2}$. The solution of this equation, unique up to solutions of the homogeneous equation, is

$$
f=-4 p \ln \rho^{2},
$$

which agrees precisely with Aichelburg and Sexl [1] (cf. eqs. (2) and (A.26)).

For a sourceless plane wave in Minkowski space we set $p=0$ to obtain

$$
\Delta f=0 \text {, }
$$

which agrees with Penrose [2].

For a spherical wave in Minkowski space we write the metric in the form

$$
\mathrm{d} \hat{s}^{2}=-\mathrm{d} u \mathrm{~d} v+\frac{1}{4}(v-u)^{2}\left(\mathrm{~d} \theta^{2}+\sin ^{2} \theta \mathrm{d} \varphi^{2}\right),
$$

so that

$$
\begin{aligned}
& A=-\frac{1}{2}, \\
& g=r^{2}={ }_{4}^{1}(v-u)^{2} .
\end{aligned}
$$


But the derivatives of $g$ are not identically zero at $u=0$. Thus, there are no spherical waves (of this form) in Minkowski space.

Physically this might seem mysterious because one expects spherical shock waves to arise in, e.g., the debris of a violent explosion. On closer inspection one concludes that there must be non-zero curvature behind such shock waves. However, note that Penrose [2] does exhibit the existence of sourceless spherical shock waves in Minkowski space but having a different form than our ansatz (eq. (B.2)).

We now turn to a more interesting example, namely the Schwarzschild metric which in (null) Kruskal-Szekeres coordinates takes the form

$$
\mathrm{d} \hat{s}^{2}=-\frac{32 m^{3}}{r} \mathrm{e}^{-r / 2 m} \mathrm{~d} u \mathrm{~d} v+r^{2}\left(\mathrm{~d} \theta^{2}+\sin ^{2} \theta \mathrm{d} \varphi^{2}\right),
$$

so that

$$
\begin{aligned}
& A=-\frac{16 m^{3}}{r} \mathrm{e}^{-r / 2 m} \\
& g=r^{2} .
\end{aligned}
$$

$r$ is given implicitly as a function of $u$ and $v$ by

$$
u v=-\left(\frac{r}{2 m}-1\right) \mathrm{e}^{r / 2 m}
$$

so that all $v$-derivatives of $r$ are proportional to $u$. Thus, the conditions on the metric coefficients $A$ and $g$ are satisfied at $u=0$.

Furthermore, since $g_{\text {, } u v} \equiv A$ the condition on $f$ becomes

$$
\begin{aligned}
\Delta f-f & =\left.32 \pi p g A\right|_{u=0} \delta(\theta) \\
& =-2 \pi \kappa \delta(\theta),
\end{aligned}
$$

where $\kappa=2^{9} \mathrm{~m}^{4} \mathrm{pe} \cdot \mathrm{I}^{-1}$ and where we have arranged the coordinates so that the photon is at $\theta=0=u$.

We now solve eq. (12) by expanding $f$ in terms of spherical harmonics $Y_{l m}(\theta, \varphi)$. We see immediately that only spherical harmonics with $m=0$ contribute; expressing these in terms of the Legendre polynomials $P_{l}(x)$ leads to

$$
f=\kappa \sum_{l} \frac{l+\frac{1}{2}}{l(l+1)+1} P_{l}(\cos \theta) .
$$

We can obtain an integral expression for $f$ by using the generating function for the Legendre polynomials, namely

$$
\sum_{t=0}^{\infty} P_{l}(x) t^{\prime}=\left(1-2 x t+t^{2}\right)^{-1 / 2}
$$


and the fact that

$$
\int_{-\infty}^{0} t^{\prime} \mathrm{e}^{s / 2} \cos \left(\frac{1}{2} \sqrt{3} s\right) \mathrm{d} s=\frac{l+\frac{1}{2}}{l(l+1)+1},
$$

where $t=e^{s}$, to finally obtain

$$
f=\kappa \int_{0}^{\infty} \frac{\sqrt{\frac{1}{2}} \cos \left(\frac{1}{2} \sqrt{3} s\right)}{(\cosh s-\cos \theta)^{1 / 2}} \mathrm{~d} s .
$$

We have not attempted to perform the integration explicitly. We note that the homogeneous equation (eq. (12) with $p=0$ ) has no solution. In the limit of small $\theta$ eq. (15) in appropriate coordinates reduces to eq. (8), with a well-determined value of the integration constant.

\section{Discussion}

The surprisingly simple geometric shape of a gravitational shock wave of massless particles in flat space can help us obtain a better understanding of gravitational interactions among particles at extreme energies. It is easy to argue that at extremely high energies interactions due to this shock wave will dominate over all quantum field theoretic interactions, simply because the latter will be postponed by an infinite time shift (due to the logarithmic singularitity in eq. (2), see fig. 4). This implies that cross sections at such energies will be entirely predictable.

A problem arises if two such particles are considered, both accompanied by their shock waves, that meet and collide. The result of such a collision will be curved shock waves which obey the vacuum Einstein field equations only if space-time after the collision in the region between both shock waves is curved, so that we then have to deal with the full complexity of general relativity. We have here a limiting case of the general problem of black hole encounters which has been studied in detail by D'Eath [4] and Curtis [5].

On physical grounds the Schwarzschild result, eq. (13), should not be surprising. The flat space result (e.g. eq. (8)) can be obtained [1] by infinitely boosting a (massive) source particle. Now take an $r=$ const observer in the usual Schwarzschild coordinates. Put a (nearly) massless particle at the horizon and wait. The observer will see a particle with an increasingly large boost! It is only natural to expect a similar result in both cases. The spherical nature of the wave in the Schwarzschild case (as opposed to the plane wave in Minkowski space) is merely a reflection of the spherically symmetric nature of the "boost" relating an $r=$ const observer to Kruskal-Szekeres coordinates. Physically, one expects any (weak) plane wave approaching the black hole to become gradually more spherical, as seen by an outside observer, as it comes closer to the horizon.

Returning to the picture of a particle of small mass falling into the black hole (see discussion after fig. 1) one expects a small increase of the Schwarzschild radius 
of the black hole, together with a slight expansion of its furture horizon. This expansion then grows exponentially with the Schwarzschild time coordinate. This is what our "shock wave" here actually describes. Eq. (15) is in closed form the extent of the horizon expansion. One might speculate what effect this expansion has on the quantum nature of the vacuum and, in particular, Hawking radiation. We believe that the gravitational interaction between infalling matter and Hawking radiation, crucial for a deeper understanding of the quantum properties of black holes themselves [6], should be described using our expression for the horizon expansion.

Finally, we are aware of the analogy with the electric field of a charged particle moving at the speed of light, which is similar to the gravitational field described here. Our gravitational shock wave can be compared to a limiting case of Cherenkov radiation.

We thank Paul Shellard for bringing the work of D'Eath [4] to our attention, which then led us to the previous work of Aichelburg and Sexl [1] and Penrose [2].

The computer calculations of the Ricci tenser were performed while one of us (T.D.) was a visitor at Queen Mary College, London. He is deeply indebted to Malcolm MacCallum and Gordon Joly for hospitality and assistance.

\section{Appendix A}

\section{A PHOTON IN MINKOWSKI SPACE}

Consider the linearized field of a point mass in Lorentz gauge:

$$
\mathrm{d} s^{2}=-\left(1-\frac{2 m}{R}\right) \mathrm{d} T^{2}+\left(1+\frac{2 m}{R}\right)\left(\mathrm{d} x^{2}+\mathrm{d} y^{2}+\mathrm{d} Z^{2}\right),
$$

with $m \& R$. This is the field of the particle as seen in its rest frame. Boost this rest frame with respect to coordinates $(t, x, y, z)$ via

$$
\begin{aligned}
& T=t \cosh \beta-z \sinh \beta, \\
& Z=-t \sinh \beta+z \cosh \beta,
\end{aligned}
$$

and simultaneously set

$$
m=2 p \mathrm{e}^{\beta}
$$

for some constant $p>0$.

Introduce null coordinates

$$
\begin{aligned}
& u=t-z, \\
& v=t+z .
\end{aligned}
$$


The momentum of the particle is

$$
p^{a}=m\left[(\cosh \beta) \delta_{t}^{a}+(\sinh \beta) \delta_{z}^{a}\right],
$$

and thus

$$
\lim _{\beta \rightarrow \infty} p^{a}=p\left(\delta_{t}^{a}+\delta_{z}^{a}\right)=2 p \delta_{v}^{a} ;
$$

in the limit the particle is massless and moves (at the speed of light) in the $v$-direction; $p$ is its momentum and is kept finite (possibly large).

Writing the metric in $(u, v, x, y)$ coordinates we obtain

$$
\mathrm{d} s^{2}=\left(1+\frac{2 m}{R}\right)\left[-\mathrm{d} u \mathrm{~d} v+\mathrm{d} x^{2}+\mathrm{d} y^{2}\right]+\frac{4 m}{R}\left[\frac{p}{m} \mathrm{~d} u+\frac{m}{4 p} \mathrm{~d} v\right]^{2},
$$

with

$$
R^{2}=x^{2}+y^{2}+\left(\frac{p}{m} u-\frac{m}{4 p} v\right)^{2}
$$

The key idea is to notice that

$$
\lim _{\substack{m \rightarrow 0 \\(u \neq 0, v, \times, y) \text { fxed }}} \mathrm{d} s^{2}=-\mathrm{d} u\left(\mathrm{~d} v-4 p \frac{\mathrm{d} u}{|u|}\right)+\mathrm{d} x^{2}+\mathrm{d} y^{2},
$$

which is flat although the coordinate $v^{\prime}$ satisfying

$$
\mathrm{d} v^{\prime}=\mathrm{d} v-\frac{4 p \mathrm{~d} u}{|u|}
$$

suffers a discontinuity at $u=0$ due to the absolute value sign. To make this somewhat more precise, introduce coordinates $(\hat{u}, \hat{v}, x, y)$ by*

$$
\begin{aligned}
& \hat{u}=u+\frac{m^{2} Z \ln (2 R)}{p R}, \\
& \hat{v}=v+\frac{4 p Z \ln (2 R)}{R} .
\end{aligned}
$$

Note that $(\hat{u}, \hat{v}, x, y)$ is obtained from $(u, v, x, y)$ by adding $(4 Z \ln (2 R) / R) p^{a}$. Then

$$
\begin{gathered}
R^{2}=x^{2}+y^{2}+\left(\frac{p}{m} \hat{u}-\frac{m}{4 p} \hat{v}\right)^{2}, \\
\lim _{\substack{m \rightarrow 0 \\
(u \times 0, v, x, y) \text { fixed }}} \mathrm{d} s^{2}=-\mathrm{d} \hat{u} \mathrm{~d} \hat{v}+\mathrm{d} x^{2}+\mathrm{d} y^{2} .
\end{gathered}
$$

* The motivation for these coordinates is as follows: in the limit, $Z / R$ acts like a $\theta$-function and reproduces the effect of the absolute value sign, while $\mathrm{d} R / R=\mathrm{d} u / u$. Furthermore, $\ln R$ is finite at $u=0$. The factor 2 is chosen for convenience. We use geometric units in which $G=c=h=1$; all quantities are dimensionless. 
The metric (A.13) is flat. It remains to investigate its behavior near $u=0$, which, in the limit, is just $\hat{u}=0$. To do this we consider the behavior of (null) geodesics crossing $u=0$ for $m \neq 0$ and then take the limit as $m$ goes to zero*. We do this both in a "near" region, which collapses to $\hat{u}=0$ in the limit - this is just the rest frame of the particle - and a "far" region, where $\hat{u}$ remains non-zero in the limit.

The (linearized) geodescis of the metric (1) are given by

$$
\begin{gathered}
\dot{T}=E\left(1+\frac{2 m}{R}\right), \\
y \dot{Z}-Z \dot{y}=L\left(1-\frac{2 m}{R}\right), \\
\dot{y}^{2}+\dot{Z}^{2}=-M^{2}\left(1-\frac{2 m}{R}\right)+E^{2},
\end{gathered}
$$

where the dot denotes derivatives with respect to the affine parameter $\lambda$ along the geodesic. We have assumed $x=0$ without loss of generality; the constants $E, L, M$ denote the energy, angular momentum, and rest mass of the test particle, respectively.

In what follows we consider only "null" geodescis; i.e. we set $M=\mathrm{O}\left(m^{2}\right)$. Expanding $y, Z$ and $T$ in powers of $m$ and considering only the terms linear in $m$ we have

$$
\begin{aligned}
& y=y_{0}+m y_{1}, \\
& Z=Z_{0}+m Z_{1}, \\
& T=T_{0}+m T_{1},
\end{aligned}
$$

and eqs. (A.14) now become

$$
\begin{gathered}
\dot{T}_{0}=E, \\
\dot{y}_{0}^{2}+\dot{Z}_{0}^{2}=E^{2}, \\
y_{0} \dot{Z}_{0}-Z_{0} \dot{y}_{0}=L, \\
\dot{T}_{1}=2 E / R_{0}, \\
\dot{y}_{0} \dot{y}_{1}+\dot{Z}_{0} \dot{Z}_{1}=0, \\
y_{0} \dot{Z}_{1}-Z_{1} \dot{y}_{0}+y_{1} \dot{Z}_{0}-Z_{0} \dot{y}_{1}=-\frac{2 L}{R_{0}},
\end{gathered}
$$

where $R_{0}^{2}=y_{0}^{2}+Z_{0}^{2}$.

\footnotetext{
* Penrose and MacCallum [7] describe some properties of such geodesics without actually calculating them. Penrose and Curtis have performed similar calculations of null geodesics crossing a shock wave, but as far as we know these have not been published [8].
} 
However, since

$$
\begin{aligned}
& u=\frac{m}{2 p}(T-Z), \\
& v=\frac{2 p}{m}(T+Z),
\end{aligned}
$$

we must require

$$
\dot{Z}_{0}=-\dot{T}_{0} \equiv-E
$$

if $\dot{v}$, and thus $\dot{\hat{v}}$, is to remain finite in the limit as $m$ goes to zero. The second and third of eqs. (A.16) now yield

$$
\begin{aligned}
& \dot{y_{0}}=0, \\
& y_{0}=-L / E,
\end{aligned}
$$

and the fifth of eqs. (A.16) implies

$$
\dot{Z}_{1}=0 \text {. }
$$

Thus

$$
\begin{aligned}
& \dot{u}=\frac{m}{p} E+\frac{m^{2}}{p} \frac{E}{R_{0}}, \\
& \dot{v}=4 p \frac{E}{R_{0}} .
\end{aligned}
$$

Using eq. (A.18) these can be integrated directly to give

$$
\begin{aligned}
& u=\frac{m E}{p} \lambda-\frac{m^{2}}{p} \ln \left(Z_{0}+R_{0}\right), \\
& v=-4 p \ln \left(Z_{0}+R_{0}\right),
\end{aligned}
$$

where we have ignored an irrelevant integration constant, and thus

$$
\begin{aligned}
& \hat{u}=\frac{m E}{p} \lambda+\frac{m^{2}}{p}\left[\frac{Z_{0} \ln \left(2 R_{0}\right)}{R_{0}}-\ln \left(Z_{0}+R_{0}\right)\right], \\
& \hat{v}=4 p\left[\frac{Z_{0} \ln \left(2 R_{0}\right)}{R_{0}}-\ln \left(Z_{0}+R_{0}\right)\right] .
\end{aligned}
$$

We now separate into a near region $\mathrm{N}$ and a far region $\mathrm{F}$ as follows:

$$
\begin{aligned}
& N=\{|\lambda|<1 / \sqrt{m}\}, \\
& F=\{\sqrt{m} \leqslant m|\lambda|<\infty\} .
\end{aligned}
$$


Note further that

$$
\begin{aligned}
& \lim _{\lambda \rightarrow-\infty} \hat{v}=0, \\
& \lim _{\lambda \rightarrow+\infty} \hat{v}=-4 p \ln y_{0}^{2}, \\
& \lim _{\lambda \rightarrow+\infty} \hat{u}=\frac{m E}{p} \lambda .
\end{aligned}
$$

Thus, there is a total shift in $\hat{v}$ given by

$$
\Delta \hat{v}=-4 p \ln y_{0}^{2} .
$$

Note that, in the limit as $m$ goes to zero, $\lambda$ is infinite everywhere in F. Furthermore, in this limit $\hat{u}$ is identically zero in $N$, whereas $\hat{u}$ is a good affine parameter in $F$ along the geodesic.

The shift (A.26) thus occurs, for small $m$, "essentially" only in N! Thus, in the limit as $m$ goes to zero, the shift (A.26) occurs at $\hat{u}=0$ and represents a finite discontinuity in $\hat{v}$ along null geodesics! This can also be seen by calculating

$$
\lim _{\lambda \rightarrow \pm x} \dot{\hat{v}}=0
$$

thus showing that in the limit as $m$ goes to zero $\hat{v}$ is constant in $\mathrm{F}$, i.e. for nonzero $\hat{u}$. This is just a reflection of the fact that, in the limit, $F$ is flat. This is illustrated in fig. 2.

We now turn to the behaviour of $y$. We must solve the last of eqs. (A.16), which, on inserting eqs. (A.19) and (A.20) becomes

$$
y_{1} \dot{Z}_{0}-Z_{0} \dot{y}_{1}=-2 L / R_{0}
$$

The homogeneous equation clearly has the solution

$$
y_{1}^{h}=A Z_{0}
$$

for any constant $A$; it remains to find a particular solution.

Multiplying eq. (A.28) by $\dot{Z}_{0}$ yields

$$
E^{2} y_{1}-R_{0} \dot{R}_{0} \dot{y}_{1}=2 L E / R_{0} \text {. }
$$

But noticing that

$$
\dot{R}_{0}^{2}=\frac{Z_{0}^{2} E^{2}}{R_{0}^{2}} \equiv E^{2}\left(1-\frac{y_{0}^{2}}{R_{0}^{2}}\right)
$$

suggests an ansatz for $y_{1}$ as a power series in $R_{0}$. We thus obtain the particular solution

$$
y_{1}^{p}=\frac{2 L}{y_{0}^{2} E} R_{0} \equiv-\frac{2 R_{0}}{y_{0}} .
$$


The general solution to eq. (A.28) is thus

$$
y_{1}=-\frac{2 R_{0}}{y_{0}}+A Z_{0},
$$

and therefore

$$
y=-\frac{L}{E}+m\left[-\frac{2 R_{0}}{y_{0}}+A Z_{0}\right] .
$$

We are interested in the behaviour of $y$ in the far field $F$ for $m$ small. We obtain

$$
\lim _{\substack{m \rightarrow 0 \\ \hat{u} \neq 0}} \frac{\partial y}{\partial \hat{u}}=-\frac{2 p}{y_{0}} \operatorname{sgn} \hat{u}-p A .
$$

This behaviour is illustrated in fig. 3 . In general we have

$$
\cot \alpha+\cot \beta=\frac{4 p}{y_{0}}
$$

for the angles $\alpha$ and $\beta$ as defined in fig. 3 .

At this point several comments are in order. We have not considered all geodesics which cross the shock wave, but only a sufficient number to determine how to glue the two flat half-space together. That this is sufficient follows from the existence of coordinates in which the metric is in fact continuous (see appendix B).

Using the results of appendix $B$ we see that

$$
\begin{aligned}
\lim _{m \rightarrow 0} \mathrm{~d} s^{2} & =-\mathrm{d} \hat{u}\left(\mathrm{~d} \hat{v}+4 p \ln y_{0}^{2} \delta(\hat{u}) \mathrm{d} \hat{u}\right)+\mathrm{d} x^{2}+\mathrm{d} y^{2} \\
& =-\mathrm{d} u\left(\mathrm{~d} v+\frac{4 p \mathrm{~d} u}{u}(1-2 \theta(u))+4 p \ln 4 \delta(u) \mathrm{d} u\right)+\mathrm{d} x^{2}+\mathrm{d} y^{2},
\end{aligned}
$$

which of course reduce to (A.13) and (A.9) respectively for $u \neq 0$. The first of (A.37) is just the result of Aichelburg and Sexl [1] (their eq. (3.9)), but the second of (A.37) disagrees with their eq. (3.10). Although this is at first disconcerting, a more careful analysis reveals the source of the discrepancy: we have taken a limit different from theirs. This can be seen by noting that for $m \rightarrow 0, u \neq 0$, our original coordinates $(t, z)$ are related to their coordinates $(\bar{l}, \bar{x})$ by infinite scale factors.

Equivalently, note that the original Minkowski space given in $(u, v, x, y)$ coordinates is "pushed to infinity" in the resulting space-time given in $(\hat{u}, \hat{v}, x, y)$ coordinates. Specifically, $\{u \neq 0 ;|v|<\infty\}$ corresponds to $(\hat{u} \neq 0 ; \hat{v}=-(\operatorname{sgn} \hat{u}) \infty\}$, although the source located at $\{u=0 ;|v|<\infty\}$, corresponds to $\{\hat{u}=0 ;|\hat{v}|<\propto\}$. The corresponding statement for the $(\bar{l}, \bar{x}, \bar{y}, \bar{z})$ coordinates of Aichelburg and Sexl [1] would be somewhat different.

Finally, note that although we have linearized both the metric (A.1) and the geodesics (A.14) the result is in fact exact. Had we begun (as in [1]) with the exact 
Schwarzschild metric in isotropic coordinates and expanded in powers of $m$ only the linear terms we consider would have survived.

\section{Appendix B}

\section{CALCULATION OF THE RICCI TENSOR}

We start with the metric

$$
\mathrm{d} \hat{s}^{2}=2 A(u, v) \mathrm{d} u \mathrm{~d} v+g(u, v) h_{i j}\left(x^{i}\right) \mathrm{d} x^{i} \mathrm{~d} x^{\prime},
$$

which is assumed to satisfy the Einstein vacuum equations. We introduce a shock wave by keeping (B.1) for $u<0$ but replacing $v$ by $v+f\left(x^{i}\right)$ for $u>0^{\star}$ :

$$
\mathrm{d} s^{2}=2 A(u, v+\theta f) \mathrm{d} u\left(\mathrm{~d} v+\theta f_{. i} \mathrm{~d} x^{i}\right)+g(u, v+\theta f) h_{i j} \mathrm{~d} x^{i} \mathrm{~d} x^{j},
$$

where $\theta=\theta(u)$ is the usual step function. Changing to coordinates $\left(\hat{u}, \hat{v}, \hat{x}^{i}\right)$ defined by

we obtain

$$
\begin{aligned}
\hat{u} & =u, \\
\hat{v} & =v+\theta f, \\
\hat{x}^{i} & =x^{i},
\end{aligned}
$$

$$
\mathrm{d} s^{2}=2 A(\hat{u}, \hat{v}) \mathrm{d} \hat{u}(\mathrm{~d} \hat{v}-\delta(\hat{u}) f \mathrm{~d} \hat{u})+g(\hat{u}, \hat{v}) h_{i j} \mathrm{~d} \hat{x}^{i} \mathrm{~d} \hat{x}^{j},
$$

where $\delta=\delta(u)$ is the Dirac delta "function".

We note that the metric $d s^{2}$ given in (B.2) and (B.4) is in fact continuous, i.e. there exist coordinates $\left(\bar{u}, \bar{v}, \bar{x}^{t}\right)$ such that the metric coefficients are continuous. A possible choice is given implicitly by

where

$$
\begin{aligned}
& \hat{u}=\bar{u}, \\
& \hat{v}=\bar{v}+\theta \bar{f}-\frac{1}{2} \bar{u} \theta^{2} \frac{\hat{A}}{\hat{g}} h^{m n} \bar{f}_{, m} \bar{f}_{, n}, \\
& \hat{x}^{i}=\bar{x}^{i}-u \theta \frac{\hat{A}}{\hat{g}} h^{i m} \bar{f}_{, m},
\end{aligned}
$$

$$
\begin{aligned}
\bar{f} & =f\left(\bar{x}^{i}\right), \\
\hat{A} & =A(\hat{u}, \hat{v}), \\
\hat{g} & =g(\hat{u}, \hat{v}), \\
h^{i j} & =h^{i j}\left(x^{i}\right) .
\end{aligned}
$$

* This is not quite the standard ansatz $g_{a b}=(1-\theta) g_{a b}^{-}+\theta g_{a b}^{+}$. However, in the examples considered here the corresponding Ricci tensors differ at worst by a term in $R_{u u}$ proportional to $\theta(1-\theta)$, which is not physically relevant. 
However, the coordinates (B.5) are extremely unwieldy both for mathematical computations and for preserving physical intuition. We will thus proceed as follows. Noting that the coordinate transformation between $\left(\bar{u}, \bar{v}, \bar{x}^{i}\right)$ and $\left(u, v, x^{i}\right)$ coordinates is continuous, we will calculate the Ricci tensor for the metric (B.2). However since the metric (B.4) is much easier to work with, we will formally transform to $\left(\hat{u}, \hat{v}, \hat{x}^{i}\right)$ coordinates, calculate $R_{\hat{a} \hat{b}}$, and then transform back to obtain $R_{a b}$. Direct calculation yields ${ }^{\star}$

$$
\begin{aligned}
& R_{\hat{u} \hat{\imath}}=-\frac{\hat{A_{\hat{i}}}}{\hat{A}} f_{, i} \delta, \\
& R_{\hat{v} \hat{i}}=0, \\
& R_{\hat{i j}}=R_{i j}^{(2)}-h_{i j}\left[\frac{\hat{g}_{. \hat{w} \hat{L}}^{\hat{A}}}{\hat{A}}+\frac{\hat{g}_{. \hat{v} \hat{v}}}{\hat{A}} f \delta\right] \text {, } \\
& R_{\hat{u} \hat{v}}=\left(\frac{\hat{A}_{\hat{u}} \hat{A}_{\hat{v}}}{\hat{A}^{2}}-\frac{\hat{A}_{\hat{u} \hat{v}}}{\hat{A}}+\frac{1}{2} \frac{\hat{g}_{, \hat{u}} \hat{g}_{, \hat{v}}}{\hat{g}^{2}}-\frac{g_{. \hat{u} \hat{v}}}{\hat{g}}\right)+\left(\frac{\hat{A}_{, \hat{v}}^{2}}{\hat{A}^{2}}-\frac{\hat{A}_{\hat{v} \hat{v}}}{\hat{A}}-\frac{\hat{g}_{. \hat{v}} \hat{A}_{\hat{v}}}{\hat{g} \hat{A}}\right) f \delta, \\
& R_{\hat{i} \hat{v}}=-\frac{\hat{g}_{. \hat{v} \hat{v}}}{\hat{g}}+\frac{1}{2} \frac{\hat{g}_{. \hat{v}}^{2}}{\hat{g}^{2}}+\frac{\hat{g}_{. \hat{v}} \hat{A}_{\hat{v}}}{\hat{g} \hat{A}},
\end{aligned}
$$

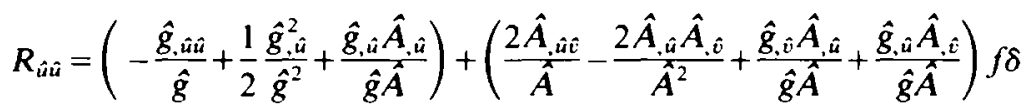

$$
\begin{aligned}
& +2\left(\frac{\hat{A}_{\hat{v} \hat{v}}}{\hat{A}}-\frac{\hat{A}_{, \hat{v}}^{2}}{\hat{A}^{2}}+\frac{\hat{g}_{.} \hat{\boldsymbol{A}}_{\hat{v}}}{\hat{g} \hat{A}}\right) f^{2} \delta^{2}+\frac{\hat{A}}{\hat{g}} \Delta f \delta-\frac{\hat{g}_{, \hat{i}}}{\hat{g}} f \delta^{\prime},
\end{aligned}
$$

where $R_{i j}^{(2)}$ is the Ricci tensor derived from $h_{i j}, \Delta$ is the Laplace operator associated with $h_{i j}$, and $\delta=\delta(\hat{u})$.

Blithely ignoring the $\delta^{2}$ term we transform to $\left(u, v, x^{\prime}\right)$ coordinates and insert the vacuum equations (obtained by setting $f=0$ ) to get

$$
\begin{aligned}
& R_{i v}=R_{\hat{i} \hat{v}}=0, \\
& R_{\mathrm{c} i}=R_{\hat{i} \hat{i}}=0 \text {, } \\
& R_{i j}=R_{i j}=-h_{i j} \frac{\hat{g}_{. \hat{v} \hat{v}}}{\hat{A}} f \delta, \\
& R_{u v}=R_{\hat{u} \hat{v}}=\left(\frac{\hat{A}_{, \hat{v}}^{2}}{\hat{A}^{2}}-\frac{\hat{A}_{\hat{i} \hat{v}}}{\hat{A}}-\frac{\hat{g}_{, \hat{i}} \hat{A}_{\hat{i}}}{\hat{g} \hat{A}}\right) f \delta, \\
& R_{u i}=R_{\hat{u} \hat{\imath}}+R_{\hat{u} \hat{v}} \theta f_{. \imath} \\
& =f_{.} \delta\left[-\frac{\hat{A}_{\hat{i}}}{\hat{A}}+\theta f\left(\frac{\hat{A}_{\hat{v}}^{2}}{\hat{A}^{2}}-\frac{\hat{A}_{\hat{v} \hat{v}}}{\hat{A}}-\frac{\hat{g}_{\hat{v}} \hat{\boldsymbol{A}}_{\hat{v}}}{\hat{\mathrm{g} \hat{A}}}\right)\right],
\end{aligned}
$$

* We note that Taub [9] has given a systematic presentation of space-times with distribution-valued curvature tensors. 


$$
\begin{aligned}
& R_{\text {uน }}=R_{\hat{\mathcal{u}} \hat{u}}+2 R_{\hat{\mathcal{u}} \hat{v}} f \delta \\
& =\frac{\hat{A}}{\hat{g}} \Delta f \delta-\frac{\hat{g}_{\hat{v}}}{\hat{g}} f \delta^{\prime}+\left(\frac{\hat{g}_{, \hat{u}} \hat{g}_{, \hat{v}}}{\hat{g}^{2}}-\frac{2 \hat{g}_{\hat{u} \hat{v}}}{\hat{g}}+\frac{\hat{g}_{, \hat{v}} \hat{A}_{\hat{u}}}{\hat{g} \hat{A}}+\frac{\hat{g}_{. \hat{u}} \hat{A}_{\hat{v}}}{\hat{g} \hat{A}}\right) f \delta .
\end{aligned}
$$

The stress-energy tensor for a massless particle located at the origin $\rho=0$ of the ( $x^{i}$ ) 2-surface and at $u=0$ is

$$
T^{a b}=4 p \delta(\rho) \delta(u) \delta_{v}^{a} \delta_{v}^{b},
$$

where $p$ is the momentum of the particle. Thus, the only non-zero component is

$$
T_{u u}=4 p A^{2} \delta(\rho) \delta(u) .
$$

Inserting (B.8) and (B.10) into the Einstein field equations, partially integrating the $\delta^{\prime}$ term, noting that e.g. $\hat{A}_{, \hat{v}}(\hat{u}=0)=0 \Leftrightarrow A_{v v}(u=0)=0$ yields precisely eqs. (5).

The calculation above was first done by hand and then checked using the algebraic manipulation computer system SHEEP. As a further check on the validity of working in the singular coordinates $\left(\hat{u}, \hat{v}, \hat{x}^{i}\right)$ (eq. (B.4)) SHEEP was also used to calculate the Ricci tensor directly in $\left(u, v, x^{i}\right)$ coordinates (eq. (B.8)), thus checking the original calculation of ' $t$ Hooft for the Schwarzschild case. The same answer, namely eqs. (5), was of course obtained in all cases.

\section{References}

[1] P.C. Aichelburg and R.U. Sexl, J. Gen. Rel. Grav. 2 (1971) 303.

[2] R. Penrose, in General relativity: papers in honour of J.L. Synge, ed. L. O'Raifeartaigh (Clarendon, Oxford, 1972) 101:

in Battelle rencontres, ed. C.M. De Witt and J.A. Wheeler (Benjamin, New York, 1968) 198; in Differential geometry and relativity, ed. M. Cahen and M. Flato (Reidel, Dordrecht, 1976) 271

[3] J. Ehlers and W. Kundt, in Gravitation: an introduction to current research, ed. L. Witten (Wiley, New York, 1962) 85f;

H.W. Brinkman, Proc. Natl. Acad. Sci. (US) 9 (1923) 1

[4] P.D. D'Eath, Phys. Rev. D18 (1978) 990

[5] G.E. Curtis, J. Gen. Rel. Grav. 9 (1978) 987, 999

[6] G. 't Hooft, J. Geom. Phys. I (1984) 45

[7] R. Penrose and M.A.H. MacCallum, Phys. Reports 6 (1973) 270

[8] P.D. D'Eath, private communication

[9] A.H. Taub, J. Math. Phys. 21 (1980) 1423 